\title{
La explicación en psicología
}

\section{Alfredo Fierro}

\author{
Universidad de Salamanca
}

En diferentes idiomas «explicación» conlleva diferentes metáforas. En castellano, asi como en el latino «explicare» ( $y$ en sus derivados: el francés "expliquer", el italiano «spiegare»), explicar da a entender una operación de desplegar o desarrollar, y también de analizar. Otro verbo latino, «explanare», del que procede la inglesa "explanation», evoca una imagen diferente, la de allanar o reducir a términos llanos la escabrosidad y obstáculos del objeto por explicar. El germano «erklären», por su lado, supone la metáfora del esclarecimiento de lo oscuro, de arrojar luz y transparencia sobre lo opaco impenetrable. Los matices idiomáticos; con todo, coinciden fácilmente en una acepción común del uso cotidiano de «explicar» en todas esas lenguas, donde queda incluido tanto el desarrollar o analizar, y el allanar, cuanto el aclarar o esclarecer.

Los epistemólogos advierten que la explicación se refiere de manera directa no a hechos o fenómenos, sino a proposiciones o enunciados. Una proposición es explicada cuando se exhiben las reglas de su necesaria derivación lógica de otra u otras proposiciones (Hempel, 1965; Braithwaite, 1965; Bunge, 1969). Las operaciones explicatorias, por tanto, tienen que ver con unidades del discurso científico, con enunciados de la ciencia, y no con sucesos del mundo real. Pese a esta advertencia terminológica, es, sin embargo, práctica científica habitual referirse directamente a la explicación de dichos sucesos, omitiendo la cautela de puntualizar que a los eventos no podemos referirnos sino mediante enunciados y que son éstos, antes que aquéllos, los que en rigor piden ser explicados y los que la ciencia trata de explicar.

Cumplida la anterior puntualización, una de las mejores definiciones posibles es la propuesta por Mauss (editado en 1970, pero escrito en 1909): «Explicar es establecer un orden racional entre los hechos». Aquí no se especifica qué es racionalidad ni tampoco cuál es el orden desde ella establecido. Pero justo esta imprecisión es la que conviene a la genérica indeterminación y a la pluralidad de acepciones de la explicación en ciencia. Hay tantos modos de explicar cuantos modos de ordenar racio- 
nalmente los hechos, o los enunciados relativos a ellos. La explicación - he aquí otra manera de declarar la misma idea - es la respuesta a la pregunta por el «por qué». Ahora bien, como a esta pregunta cabe dar respuestas de muy diverso orden, hay tantos modos de explicación cuantos órdenes de posible respuesta al «por qué». O, para decirlo todavía de otra forma, puesto que explicar un hecho es enunciar la razón suficiente del mismo, los modos de explicación son tantos como los modos de razón.

La explicación en ciencia se halla estrechamente vinculada a la formulación de leyes, o, más bien, de «enunciados legaliformes», por decirlo en un lenguaje epistemológico más riguroso (Bunge, 1969). Allí donde en psicología, o en otra ciencia, haya búsqueda y hallazgo de enunciados con forma de ley, allí podemos considerar (contra la opinión de Piaget, 1963, 1970, y de Greco, 1967) que hay explicación o, al menos, comienzo y rudimento de ella. Pertinentes para una reseña de los conceptos varios de explicación en psicologia serán también, por ello, las diversas concepciones acerca de la naturaleza de las leyes psicológicas. Sin embargo, como la introducción del concepto epistemológico de ley no aporta nada especifico a la tipificación de los modos de explicación en psicología, en este trabajo, bajo la rúbrica de tipos de explicación psicológica, quedan recogidos asimismo los tipos de enunciados legaliformes propuestos en psicología.

Hay algunas exposiciones sistemáticas de la multiplicidad de formas de explicación en psicologia. Debemos a Piaget (1963) una de las más completas, exponiendo y sistematizando siete diferentes tipos de explicación causal de los fenómenos psíquicos; e igualmente sobresale en su alcance sistematizador la de Spence (1948), posteriormente enriquecida por Madsen (1959), basada no en el concepto de explicación, sino en la clasificación de las leyes psicológicas de acuerdo con el género de variables cuya regular relación enuncian. Castorina y otros (1973) han abordado también de modo sistemático el tema de la explicación en psicología, declarando muy explícitamente su nexo con premisas epistemológicas. El tema de la explicación - y el de las leyes - no está del todo ausente de la literatura psicológica más actual, como se verá a lo largo de este informe, pero, desde luego, tampoco se halla en primer plano de actualidad ni mucho menos. Buena ilustración de ello la tenemos en el dato de que la tópica de los Psycbological Abstracts no contiene una entrada ni para «explication» ni para «explanation». Desde luego, para una presentación sistemática de los modos de explicar los fenómenos conductuales, hace falta inevitablemente recurrir a formulaciones teóricas que no son de hoy ni de ayer, aunque sí son clásicas y continúan determinando las suposiciones, a menudo implícitas, de hoy.

Me propongo desarrollar de manera esquemática una tipología de los modos de explicación psicológica actualmente en uso o, por lo menos, no en completo desuso. No sigo a Spence y Madsen en el criterio tipificador, puesto que no hago la caracterización sobre la base de las variables cada vez en juego, lo que no quita a que algunas de las relacio- 
nes entre éstas (en particular, las relaciones E-R, y las R-R) sí que dan lugar a algunos de los tipos por mencionar (el tipo 4 y el tipo 5, respectivamente). El criterio de mi tipificación se asemeja más bien al de Piaget, centrado, como está, en la naturaleza del «explanans» o «explicans», aunque distribuyendo las clases de «explicatorios» de modo diverso al suyo. La idea directriz es que en psicología hay un solo "explicandum», fenómeno o conjunto de fenómenos por explicar, a saber, el comportamiento, mientras que diferentes estrategias proponen alternativos «explicantia» o instancias explicativas, desde las que, por consiguiente, se delinean distintos órdenes racionales entre los hechos, diferentes géneros de respuesta al «por qué» de la conducta, dispares modos de explicación científica. De estos dispares modos, por lo demás, hago reseña, y no valoración crítica.

Comenzaré por aquellos en los que la explicación parece quedar reducida a otras dos posibles operaciones a las que, desde otras posiciones epistemológicas, y por lo general, se la suele contraponer: a la comprensión (e interpretación) y a la descripción, continuando luego por las formas de explicación estimadas más "fuertes», y propiamente tales.

\section{La explicación descriptiva}

Los antecedentes filosóficos del descriptivismo explicativo se hallan en Hume. De la crítica de Hume a la noción de causa procede la idea de que la causalidad se reduce a la sucesión o, más exactamente, a la regularidad de la sucesión entre fenómenos. El concepto escolástico de ciencia como «conocimiento a través de las causas» queda abandonado por apoyarse en la falacia del «post hoc, ergo propter hoc» («después de esto, luego a causa de esto»), y el contenido causal de la explicación científica -que hallaremos, de todos modos, en la psicología moderna- se ve reemplazado por un contenido meramente descriptivo. No hay, en rigor, conocimiento del «propter hoc», sino solamente del «post hoc». En consecuencia, explicar no puede ser otra cosa que describir sucesiones de fenómenos, poner de manifiesto relaciones regulares de anterioridad y de posterioridad entre los eventos.

En la psicología actual suele distinguirse netamente entre la descripción y la explicación de los fenómenos, y así distinguen las demás conceptualizaciones por reseñar aqui. Pero tampoco falta la versión de explicación que la hace equivaler a una descripción extensa o en un plano más elevado a una descripción en la que el fenómeno conductual por explicar se manifiesta inserto en una secuencia o cadena de fenómenos antecedentes y consecuentes tan amplia que esta inserción misma se supone valer ya por una explicación (así Eysenck, 1952, cap. I; Musso, 1970). El descriptivismo psicológico diluye la cuestión del por qué en la cuestión del cómo. En este enfoque un hecho de conducta es meramente descrito cuando es identificado, caracterizado y medido en sí mismo; y, más allá de la mera descripción, es explicado cuando se identifican, 
caracterizan y miden otros hechos, estimulares o conductuales, en cuya sucesión está integrado aquél. Explicación es entonces aquel género de descripción que toma por objeto un segmento de la realidad más amplio que aquel que pretende explicar.

\section{La explicación comprehensiva}

Tiene en común con la anterior el reducir la explicación a otra operación discursiva, ahora no a la descripción, sino a la comprensión o intelección de las conductas descritas mediante la aprehensión de un sentido del que serían portadoras. Pero de aquélla se separa muchísimo desde sus mismas raíces filosóficas. Mientras la explicación descriptiva deriva de un empirismo radical, que disuelve la causalidad en mera regularidad de las sucesiones, la explicación comprehensiva procede de una fenomenología hermenéutica, principalmente la de Dilthey, aplicada a interpretar significantes para descubrir en ellos, por debajo de ellos, los significados que alli han llegado a expresarse, a tomar cuerpo. Explicar una conducta es entonces tanto como interpretarla y llegar a aprehender su sentido, tanto el sentido que posee para el sujeto actor de ella, cuanto el sentido y mensaje que por su mediación este actor trata de hacer llegar a otras personas en una actividad conductual esencialmente significativa y comunicativa.

Es sabido que la pregunta por el sentido y la consiguiente explicación de la conducta mediante el sentido resultan del todo ajenas a la tradición conductista y, en general, a toda la psicología objetiva. Obedecen al planteamiento de otro paradigma de la psicología, el de una psicología fenomenológica, cuyas aportaciones a la teoria de la percepción («Gestalt») y a la teoria del sí mismo («self») no suelen considerarse suficientes para acreditarla con el título de cientifica. Con todo, al margen de su validez o invalidez epistemológica, el hecho es que hoy día persiste y se halla aún en circulación la idea de que explicar una conducta consiste en interpretarla y comprenderla. Hay más: la ortodoxia de la psicología fenomenológica entiende que la psicología constituye una ciencia no explicativa, sino comprensiva y hermenéutica, una ciencia que interpreta y trata de comprender el sentido de los actos humanos, mas no de explicarlos o referirlos a algún género de determinación (Jaspers, 1913; Ellenberger, 1958; Binswanger, 1973). Lo cual no obsta, sin embargo, camo acertadamente comenta Musso (1970), a que, tras este primer rehusamiento de la explicación conductista y objetivista en nombre de la comprensión fenomenológica, no reaparezca subrepticiamente la tentativa explicatoria, en la que a la comprensión misma se le hace desempeñar un papel de explicación.

Ampliándolo no poco, el concepto de comprensión podría incluso llegar a valer por todo género de explicación no causal, género donde cabría entonces incluir la explicación mediante relaciones de implicación que Piaget $(1963,1970)$ considera típica de los fenómenos psíquicos, en 
contraste con la explicación mediante relaciones estrictamente causales, características, en cambio, a su juicio, de los fenómenos físicos y fisiológicos.

\section{Las explicaciones reduccionistas}

En ellas se intenta dar razón de fenómenos de orden conductual mediante su reducción a fenómenos de otro orden. La reducción más frecuente - aunque no la única intentada; cabe también reducir por el otro lado, hacia las ciencias sociales - ha consistido en buscarles explicación en fenómenos de carácter neurofisiológico. La existencia de una entera disciplina liminar entre uno y otro orden, la psicofisiología, ha podido favorecer el equívoco de que, en último análisis, es el neurofisiólogo quien posee el secreto y las claves explicatorias de lo que el psicólogo no pasa de conocer superficialmente. Aunque las relaciones entre psicología y neurología (en general, ciencias biológicas) se hallan perfectamente clarificadas desde hace tiempo en la teoría psicofisiológica (cf. Pribram, 1962; Ardila, 1975), no faltan las apreciaciones de que la psicología constituye una disciplina truncada y frustrante puesto que no puede alcanzar las explicaciones últimas - supuestamente fisioneurológicas - de los fenómenos que observa (asi, Crick, 1979).

La propia teoría psicológica ha dado pie a menudo a explicaciones reduccionistas. Cuando Hull $(1943,1951,1952)$ propone constructos hipotéticos, como el de «drive» o impulso, está pensando que éstos, aunque por el momento no pueden ser relacionados con ningún referente fisioneurológico inequívoco a causa de la limitación de nuestros presentes conocimientos, seguramente lo tienen, y la futura investigación neurológica podrá llegar a identificarlo. La noción hulliana de constructo hipotético contiene, en consecuencia, como bien ha advertido Tous (1978), un biés reduccionista, en virtud del cual la explicación propiamente tal de la conducta residiria en entidades y procesos estudiados no ya por la psicología, sino por la correspondiente ciencia reductora.

Se comprende perfectamente que en un proyecto coherente de ciencia autónoma de la conducta, como es el skinneriano, sólo quepa repudiar este género de explicaciones reductoras. Estas caen para Skinner (1959; original de 1950) dentro del concepto -en su contexto, despectivode "teorias", de las que puede, a su juicio, prescindir por entero una ciencia de la conducta y del aprendizaje. La definición skinneriana a este propósito cuadra como anillo al dedo al reduccionismo fisioneurológico: teoría es «cualquier explicación de un hecho observado que apela a acciones que se producen en otro lugar, a otro nivel de observación, descrito con términos diferentes y acaso medido de acuerdo con otras dimensiones». ¿Son, en este sentido, necesarias las teorias del aprendizaje? La respuesta de Skinner es redondamente negativa. El conocimiento científico del aprendizaje, o de la conducta en general, para nada necesita - contra la suposición de Hull - acudir a constructos hipotéticos cuya 
final observación, medición y manipulación correspondan a ciencias diferentes de la psicologia. Una ciencia autónoma de la conducta, es decir, una psicologia, ha de construirse sobre explicaciones propias (cf. de nuevo Tous, 1978) y no sobre explicaciones reductoras a otro orden de procesos, explicaciones que resolverian y disolverían el discurso psicológico en neurofisiología.

\section{La explicación causal-funcional}

El principio de razón suficiente y no necesariamente el de causalidad subyace a toda operación explicativa. A veces, sin embargo, un principio de causalidad se halla también positivamente implicado. Algunas de las explicaciones que estamos considerando, $y$, por supuesto, la recién considerada, son de índole causal. Con todo, frente a la explicación reduccionista, la preconizada en la más pura línea conductista merece con mejor título el nombre de causal porque busca para los hechos de conducta causas (eficientes, no formales o, mucho menos, finales, teleológicas) en el orden mismo psicológico, y no en otro orden reductor, supuestamente más básico. En la explicación conductista descuella de modo singular la causalidad eficiente. Es un análisis causal que busca y asigna causas o determinantes psicológicos - a saber, los estímulos - para los efectos de conducta o de respuesta. Lo de "causal» ha de entenderse, de todas formas, en su acepción vulgar, no metafisica; y no por fuerza envuelve algún compromiso con la noción filosófica de causalidad. Debe entenderse sencillamente como determinación de unos fenómenos (los «efectos» conductuales) por otros (las «causas» estimulares), como dependencia de aquéllos con respecto a éstos.

En psicología esta concepción causal de la explicación anda aparejada al método experimental, en el que se presume que las variables independientes, o estimulares, directamente manipuladas por el experimentador, constituyen «causas» o determinantes de las variables dependientes, o conductuales, que son el resultado de aquéllas y de su manipulación. Precisamente la ventaja crucial que suele reconocerse a la experimentación frente al análisis correlacional, pero sólo observacional, es que en ella, a diferencia dè éste, se estima posible la identificación directa de nexos de causalidad o determinación (cf. Aronson y Carlsmith, 1968). El esquema metodológico experimental de variable independiente/dependiente se ve duplicado y reproducido en el esquema teórico de estímulo/respuesta; y, en este doble esquema, el primer término, determinante, pasa por ser explicativo del segundo término, determinado y explicado (cf. Bowers, 1973).

Como el léxico de la causalidad se considera muy lastrado de carga metafísica, de este género de explicación suele hablarse más bien como explicación o análisis funcional, y no causal. Un análisis funcional define la conducta como variable dependiente de otras variables susceptibles de descripción en términos físicos, consistentes en energías que influyen 
en el organismo agente desde el exterior; se propone, por tanto, explicar los hechos conductuales señalando de qué condiciones estimulares (elicitantes, señalizadoras y de refuerzo) es la conducta función (Skinner, 1953; Sidman, 1960; Barret, 1977; Silva, 1980).

He asimilado el análisis funcional de la conducta a una explicación causal o mediante eventos determinantes, pero no faltan las declaraciones, incluso en el propio Skinner, en las que el nexo funcional parece quedar interpretado en el sentido de una mera sucesión (de acuerdo con el tipo 1 de explicación descriptiva) o acaso de una covariación (según el tipo 5 de explicación correlacional). Transcribo una declaración de este tenor: «La antigua relación causa-efecto se convierte en una relación funcional. Estos nuevos términos no indican cómo la causa produce su efecto; se limitan simplemente a afirmar que hechos diferentes tienden a producirse juntos en un cierto orden" (Skinner, 1953; trad. cast. pág. 53). Afirmación tan ambigua deja al análisis funcional en posición indecisa ante tres de los tipos de explicación aquí expuestos: el descriptivo, el causal y el correlacional. Veamos ahora cómo se define este último cuando propiamente se contrapone al anterior.

\section{La explicación correlacional}

En psicología goza de buen arraigo este género de explicación en contraste al causal-funcional, y en respectivo estrecho parentesco con las dos disciplinas de la psicología científica (Cronbach, 1957). Los métodos comparativos, correlacionales, multivariados, factoriales, se ordenan al hallazgo de estructuras latentes que, en una parsimonia de dimensiones o factores respecto a las conductas manifiestas, dan razón de la varianza de éstas y, en ese sentido, las explican. El tipo de explicación y de leyes explicativas es ahora R-R y no ya E-R. El experimentalismo psicológico supone que sólo la manipulación experimental permite establecer leyes de determinación y nexos verdaderamente explicativos, mientras la investigación $\mathrm{R}-\mathrm{R}$ no pasa del descubrimiento de regularidades de correspondencia o covariación, sin valor explicatorio. Por su lado, los investigadores de orientación correlacional, y no estrictamente experimental, afirman la neta superioridad de los métodos multivariados sobre los univariados o de experimentación (Cattell, 1959, 1966), y ponen el mayor énfasis en la circunstancia de que, sin aguardar a los psicólogos experimentadores, la naturaleza está experimentando desde siempre y es en los experimentos de ella misma donde mejor y más de prisa podemos llegar a conocerla (Miller, 1977; Bronfrenbrenner, 1979).

En el contexto de una psicologia correlacional, Galanter (1970) llega a aseverar que las leyes «causales», E-R, son más bien tecnológicas o de ingenieria conductual, y no, en rigor, cientificas. A su modo de ver, las causas estimulares tienen entrada en la psicología propiamente como parámetros y no como variables. Las variables implicadas en las leyes de la psicología o ciencia de la conducta han de ser todas ellas psicológicas 
o conductuales. Lo que conduce, obviamente, a Galanter a la tesis de que dichas leyes deben ser del tipo R-R y no E-R. En consecuencia, la explicación psicológica no está ya en los estímulos, en la causalidad o determinación por parte de algunas variables independientes y antecedentes; reside, en cambio, en el círculo completo de las leyes R-R, de los lazos entre variables de conducta o de respuesta. Explicar una conducta es, en esta conceptuación, situarla en una red nomológica (Cronbach y Meehl, 1955) de proposiciones que enuncian las regularidades de su relación con otras conductas o fenómenos psiquicos.

\section{La explicación genética}

Hasta cierto punto toda teoría que se aplica a dar razón de las conductas invocando los procesos de su aprendizaje constituye, ya una explicación genética. Si explicar una conducta es referir los procesos mediante los cuales ha sido adquirida o aprendida, el aprendizaje pasa a representar un verdadero principio explicativo de la conducta no innata; y, puesto que aprendizaje de adquisición es tanto como formación, construcción o genésis de conductas nuevas como resultado de la experiencia, la explicación por el aprendizaje merece, desde luego, la denominación de constructivista o genética. Cuando "psicología del aprendizaje» no es un área de la psicología entre otras, sino la psicología a secas, la entera ciencia de la conducta bajo un determinado prisma, el de dar cuenta de ella en términos de aprendizaje (cf. Pelechano, 1980, pág. 30), nos hallamos ante un modelo explicativo que, si bien puede hallarse entrecruzado con un análisis funcional, representando hasta ahi una variedad de nuestro tipo 4, en realidad y en rigor ya no es causal o funcional, pues sólo con alguna impropiedad podría sostenerse que el aprendizaje sea causa o determinante de la conducta aprendida, y no más bien su origen o su génesis.

Las explicaciones genéticas, hallan, sin embargo, su mejor caldo de cultivo en el punto de vista evolutivo, en la contemplación del desarrollo del individuo y de las especies. Su versión más difundida en psicologia evolutiva es la de Piaget $(1950,1971)$. La explicación piagetiana de los fenómenos psíquicos es, ante todo y esencialmente, una explicación por su génesis, por el proceso de sucesivas construcciones de esquemas operatorios que han conducido al sujeto hasta su estructura actual de adaptación al entorno. La psicología evolutiva piagetiana se presenta repetidas veces bajo la explícita metáfora de una embriologia de la conducta. En el constructivismo genético e histórico de Piaget (1968), y a diferencia del estructuralismo en boga durante los años 60 , toda clase de estructuras, pero muy en particular las estructuras conductuales, los esquemas operatorios del sujeto; son el resultado de unos procesos de construcción (lo que dicho sea de paso, resolvería el dilema y la polémica de Sartre, 1960, y Lévi-Strauss, 1962: o estructura o historia), procesos que constituyen la clave explicatoria de las conductas efectivas. 
En su vertiente formal, en su formato epistemológico, y aunque aplicadas a fenómenos distintos, la psicología de Piaget y la de Freud se muestran perfectamente isomórficas en el género de explicación, psicogenética, que postulan para comportamientos actuales. Otras explicaciones en psicología evolutiva aparecen no sólo genéticas, pretendiendo dar cuenta de unas conductas en la génesis de su construcción, sino propiamente ontogenéticas, apelando a algún principio de maduración individual, como la epigénesis de Erikson (1968) o la ortogénesis de Werner (1957). Ahora bien, en cuanto comenzamos a remontarnos hacia la génesis para explicar fenómenos actuales, la búsqueda de los origenes puede no tener ya término, y desde luego no lo tiene en la ontogénesis. Dentro de las ciencias biológicas, se han constituido nuevas disciplinas de orientación evolutiva, en particular la genética de la conducta y la etología (sobre sus enseñanzas para la psicología, cf. Lockard, 1971), que llevan la explicación por la génesis a otro orden, al de la filogénesis, con lo que el género de explicación ahora comentado sale ya del terreno de la psicología, viniendo a parar en el tipo 3, reduccionista, anteriormente reseñado.

Toda clase de explicación por la génesis contrasta, por lo menos a primera vista, con el principio, vehementemente sostenido por Lewin, de que los determinantes de la conducta deben ser contemporáneos a ella, presentes, actuales, y de que en ningún modo podemos buscarlos en un pasado que no existe ya, que carece de realidad y que, por consiguiente, de ninguna manera puede influir en la determinación de fenómenos conductuales de ahora mismo. Sin embargo, la discrepancia de fondo entre las explicaciones genéticas de la conducta y las explicaciones de acuerdo con un principio de contemporaneidad seguramente es mucho más reducida de lo que al primer pronto podría sospecharse. Por un lado, la explicación por la génesis en absoluto imagina al pasado influyendo de manera mágica sobre el presente; más bien, sin violar el principio de contemporaneidad, considera que lo determinante no es el pasado como tal, sino las huellas persistentes y actuales del pasado, cualesquiera sean tales huellas: patrimonio genético, patrones específicos de conducta, aprendizajes, memoria o esquemas operatorios. Por otro lado, el propio Lewin (1951) excluye explicitamente que dicho principio sea incompatible con el de una psicología evolutiva e histórica, aplicada, en general, a señalar relaciones genéticas de determinación. Seguramente compatibles con otros modos explicativos que se ciñen a un análisis de relaciones en sincronía, las teorias genéticas resaltan la dimensión de temporalidad, de desarrollo y de extensión diacrónica, que, desde luego, caracteriza a los organismos vivos y, mucho más, al hombre. El énfasis en la diacronía, y en la génesis diacrónica de los fenómenos de conducta, separa a estas teorías de todas las demás, sólo sincrónicas, aunque, por otra parte, deje abierta la posibilidad de composición con ellas. 


\section{La explicación mediante modelos}

Modelo vale aquí por cualquier sistema, ya concreto, ya formal, que sirve para representar e ilustrar unas hipótesis teóricas acerca de relaciones entre datos empíricos. Modelo es tanto como analogía o metáfora sistemáticamente desarrollada e incorporada a una teoria científica como artificio para poner algún orden racional en sus datos. A la ciencia le son consustanciales los modelos. Gracias a ellos trata de representar sistemas y procesos peor conocidos mediante la analogía con otros sistemas y procesos mejor conocidos, o sencillamente conocidos bajo un determinado aspecto en que los primeros permanecen oscuros. La teorización mediante modelos representa un sistema como si tuviera propiedades materiales o funcionales semejantes a las de otro sistema, a las del modelo; constituye siempre, por eso, una simulación en el más amplio sentido de este término, y también una idealización, o construcción de un objeto o fenómeno ideal, esquemáticamente representativo de la realidad empírica, pero diferente de ésta (Miller, 1955; Bunge, 1972).

Puesto que la adopción de modelos sirve para establecer un orden racional entre los fenómenos en ellos simulados o modelizados, la modelización por sí sola representa ya un modo de explicación. Buena parte de las explicaciones científicas son pura y simplemente explicaciones por simulación o mediante modelos. Según desde qué perspectiva se juzgue, esta supuesta explicación no pasa de ser una ilustración mediante ejemplos más o menos pertinentes, sustituyendo la metáfora esclarecedora a la explicación propiamente dicha (Greco, 1967; Turbayne, 1970). Pero, si bien los modelos no deparan una explicación causal, ni tampoco funcional, si que constribuyen a poner orden y racionalidad en los fenómenos, y en ello son explicatorios, hasta el extremo de que de la ciencia en general (Bunge, 1972) y de la psicología en particular (Castorina y otros, 1973) ha llegado a afirmarse que versan directa e inmediatamente acerca de modelos ideales o esquemáticos de la realidad, y sólo de manera indirecta, por mediación de éstos, acerca de la realidad misma.

Aun omitiendo otras posibles variedades de modelos - v. gr., gráfi$\cos -$, en psicología y en otras ciencias hay que distinguir, al menos, dos especies bien diferenciadas: modelos consistentes en sistemas formales, lógicos o matemáticos, asociados con teorías científicas; y modelos constituidos por sistemas materiales o reales, tan empíricos como los sistemas por explicar aunque de otro orden, de otra materialidad y sustancia. De acuerdo con esta distinción, hay dos subtipos de explicación por modelos en psicología:

\section{7a. La explicación mediante modelos matemáticos}

El entero sistema de las matemáticas puede ser considerado como un rico arsenal de modelos a disposición del científico para tratar de poner con ellos orden en la aparentemente caótica multitud de los hechos 
empíricos. Lo mismo cabe decir de la estadistica en sus diversas ramas, descriptiva e inferencial, paramétrica y no paramétrica: es un vasto conjunto de modelos útiles en psicología y en otras ciencias para representar y ordenar racionalmente los datos. La psicología matemática se especializa en el desarrollo de los modelos matemáticos particularmente idóneos para ordenar los fenómenos conductuales y para valorar - mediante las pertinentes reglas de correspondencia - la bondad del ajuste de estos modelos y funciones matemáticas a los datos empíricos observados. En sus diferentes orientaciones - teorias probabilísticas de la percepción y de la conducta, ecuaciones matemáticas para el condicionamiento y el aprendizaje, funciones psicofisicas, psicometria - lcs modelos matemáticos tienen antiguas y prestigiadas raíces en psicología; y la formalización o modelización matemática de las relaciones empíricas suele ser aceptada como una variedad de explicación, compaginable, sin duda, con otros modos de explicación posibles, pero irreductible a ellos. En particular, si bien es cierto que las teorias matemáticas del aprendizaje se han desarrollado a partir de modelos explicatorios de índole causalfuncional (tipo 4), a la postre han concluido por independizarse de este origen, haciendo recaer el peso de la explicación sobre las relaciones matemáticas mismas a las que manifiestan ajustarse las variables empíricas.

Inicialmente, y en principio, modelos estadísticos fueron introducidos en psicología en orden a la mejor descripción de los datos, a su descripción más exacta, cuantitativa, y a la representación más económica de las diferentes mediciones o cuantificaciones. Eran modelos matemáticos tomados de una estadística fundamentalmente descriptiva. Todavía puede considerarse tan sólo descriptivo el uso de fórmulas matemáticas cuando son elegidas por su mayor precisión frente a las formulaciones verbales, siempre amenazadas de ambigüedad y de equivocos (Bjork, 1973). Pero, más allá de este uso ventajoso del lenguaje matemático, en vez del verbal, en la formulación descriptiva de los datos y en la enunciación hipotética de las teorías, actualmente, los modelos estadísticos y, en general, los modelos matemáticos constituyen verdaderos instrumentos explicativos y predictivos. No son meras derivaciones o especificaciones de la teoría psicológica, sino conjuntos sintácticos de reglas que hacen posible esa derivación, especificación y, en definitiva, ampliación de la teoría a la que acompañan (Rosenberg, 1968), permitiendo derivar enunciados empíricos concretos a partir de los postulados más generales de una teoría (Arnau, 1977). Las transformaciones matemáticas mismas de una formulación inicial cualquiera generan proposiciones derivadas que pueden igualmente ser sujetas a control empírico. En tanto que conllevan sus propias règlas - estadísticas o, más generalmente, matemáticas - de inferencia probabilística o de estricta derivación, estos modelos, además, reflejan, mejor que ningún otro tipo de explicación, la naturaleza de la operación explicatoria según los epistemólogos, operación directamente relativa a la derivación de unas proposiciones científicas a partir de otras, y sólo indirectamente relacionada con los fenómenos sobre los que éstas versan. 


\section{La explicación mediante simulación en computadora}

Desde sus inicios, en el intento de comprender y explicar sistemáticamente la conducta, la psicología ha recabado analogias en otros sistemas, estudiados por diferentes ciencias, tomándolos como modelos del sistema de los procesos conductuales (cf. Rechea, 1980). Uno de los modelos de más prolongada vigencia ha sido el de la homeostasis. La psicología clásica de la motivación apeló a la regulación homeostática, característica del sistema de los equilibrios y de los cambios fisiológicos, como modelo a cuya imagen y semejanza parecía posible esclarecer los procesos conductuales dinámicos. La psicología evolutiva a menudo ha acudido a modelos filogenéticos para ordenar racionalmente los fenómenos de desarrollo ontogenético. La etologia habla -metafóricamente también, es decir, en modelo - de ciertos estímulos como "disparadores» de secuencias conductuales fijas y características de una especie animal. La psicologia social utiliza modelos de juego, y de reglas de juego, para explicar las relaciones de intercambio e interacción sea en la díada, sea en el grupo. La noción de «feedback» o «retroinformación», presente hoy en muchas áreas de la psicología, implica igualmente un modelo cuyo ejemplar elemental típico lo proporciona el termostato. Modelos fisiológicos, biológicos, mecánicos, y otros, han estado incorporados siempre, con diverso grado de presencia, a la teoría y a la explicación psicológica. La adopción de modelos semejantes supone algún género de homología o isomorfismo entre diferentes sistemas (Miller, 1955), analogia formal que se halla mediada por el sistema conceptual del teórico que elabora el modelo, y que permite relaciones de reciprocidad entre un sistema y otro, v. gr., entre el biológico y el psicológico, que pueden intercambiar sus respectivas posiciones de "modelizador» y «modelizado», según se produzca el progreso en el conocimiento y también según las necesidades presentes de la teoría y de la investigación.

Sin haber hecho desaparecer del todo a esos modelos, que todavía prestan meritorios servicios en dominios concretos de la explicación de la conducta, el modelo ahora indisputablemente dominante, omnipresente, es el del procesamiento de la información o, con mayor generalidad, el modelo cibernético. La gran analogía actual, la metáfora más fructífera para la teorización psicológica - lanzada por Turing (1950) en su provocativa pregunta: «¿Pueden las máquinas pensar?»- es la de la computadora, mejor dicho, la del programa de computadora. A diferencia de otros modelos o analogías, meramente ilustrativos o representativos, el programa de computadora constituye un modelo en acto, operativo, susceptible de manipulación experimental. Es un modelo que permite la simulación. Esta ha sido definida como «el ejercicio de una flexible imitación de procesos y resultados con el propósito de clarificar o explicar los mecanismos implicados subyacentes» (Abelson, 1968). El concepto de simulación agrega al de modelo la posibilidad de modificar las condiciones de éste a voluntad del investigador. La simulación constituye así una herramienta del método experimental y no sólo - como 
los modelos en general - un artefacto conceptual, un auxiliar de la representación teorética.

Es posible la simulación de conductas fuera de la computadora. Por ejemplo, los juegos y sus reglas son amplia y experimentalmente utilizados por psicólogos sociales como sistemas simuladores del intercambio personal y grupal. Pero la mayor parte de simulación en la psicología de hoy se hace en computadora. La tecnologia de la computadora ha traído una verdadera revolución en el armamento del psicólogo, como puede apreciarse cotejando las dos compilaciones de Sidowski (1975; Sidowski y Ross, 1969) sobre instrumentos de la investigación conductual. El irresistible ascenso de una psicología cognitiva que entiende los procesos cognoscitivos como procesamiento de la información se alza en paralelo al auge de la simulación en programas de computadora, en parte provocándolo y en parte dependiendo de él. Sin embargo, el buen congeniar entre psicología cognitiva y programas de simulación no quiere decir que estos últimos tengan limitadas sus posibilidades a la psicología de los procesos cognitivos. Aunque mucho menos desarrollados, han sido asimismo aplicados programas a simular, entre otros fenćmenos (Colby, 1978), la conducta neurótica y paranoica (Colby, 1963, 1975), procesos de intercambio social (cf. Huessmann y Levinger, 1976) y, en general, procesos de personalidad (Tomkins y Messick, eds., 1963).

En el contexto del tema que nos ocupa, el de los modos de explicación de la conducta en psicología, hay que resaltar que toda metodología de la simulación, como queda ya explícito en la definición más arriba recogida, conlleva un propósito explicativo. Simular con éxito - -se asume - es tanto como explicar. Newell, Shaw y Simon (1958) han formulado muy tempranamente y de manera bien expresa esta pretensión explicatoria: «Una explicación de una conducta observada del organismo es proporcionada por un programa de procesos de información que genera dicha conductan. Programas de computadora, como el del Teórico Lógico o el del Solucionador General de Problemas, son, en consecuencia, dentro de este modelo, verdaderas explicaciones de las correspondientes conductas cognitivas de razonamiento lógico y de resolución de problemas.

Los programas para computadora prestan así a la psicología todos estos oficios en una sola pieza: un instrumental revolucionario respecto a los instrumentos tradicionales suyos (y no olvidemos que, desde Galileo y su utilización científica del telescopio, los avances más espectaculares de la ciencia estuvieron siempre vinculados a la tecnología de nuevos instrumentos; en cierta medida, el instrumento "crea» el fenómeno); una estrategia experimental para la validación de hipótesis (la comparación de conductas reales con los resultados o «salida" tras la ejecución de un programa); un modelo teorético general de explicación de la conducta por vía de su simulación; y un lenguaje riguroso y preciso, como el propio igualmente de los modelos matemáticos, que no deja resquicios para las ambigüedades que lastran al lenguaje ordinario. 


\section{La explicación predictiva}

En realidad, es un género de explicación que no se contrapone por fuerza a los demás, salvo en el acento que coloca en que la predicción atinada constituye la piedra de toque de la explicación. Al predecir, formulamos generalizaciones y proyectamos principios sobre el futuro modo de desarrollarse determinadas relaciones observadas en el pasado. Una proyección predictiva genera proposiciones sobre la existencia o la naturaleza de fenómenos todavía no observados o no suficientemente analizados. Ahora bien, las relaciones de la predicción con la explicación, en psicología, son ellas mismas objeto de alternativas conceptualizaciones.

Por de pronto, hay una multiplicidad de sentidos en los cuales la anticipación predictiva pueda afirmarse esencial a la enunciación legaliforme propia de la ciencia (Koch, 1959). Además de esto, en ocasiones, explicación y predicción de la conducta son presentadas como operaciones netamente distintas, de suerte que el poder y exactitud predictivos de una hipótesis se consideran insuficientes para fundamentar su valor teórico y explicativo, que, en este caso, parece referirse a un orden de procesos más profundos que los empíricamente observables y predictibles (así, Cronbach, 1957, p.683; y Guthrie, 1959, p.173); o, a la inversa, de modo que la explicación, entendida entonces como exclusivamente posterior a los hechos, sería deficiente respecto a la predicción, anterior a los mismos. Otras veces - y parece una conceptualización más acepta$\mathrm{da}$ - explicación y predicción son las dos vertientes simétricas de una misma operación científica: explicamos un fenómeno cuando podemos predecir su ocurrencia en futuras circunstancias; $y$, a la recíproca, esta predicción respecto a lo todavía no ocurrido u observado sólo es posible gracias a la explicación de lo ya sucedido y observado (Kaplan, 1964).

En cualquiera de las conceptualizaciones aludidas, el enfoque ahora comentado se caracteriza por desplazar la criteriología de las hipótesis y teorías psicológicas, polarizándola en el criterio no de su valor explicatorio, sino de su poder predictivo. Este desplazamiento posee indudables ventajas metodológicas y de operacionalización; y contribuye, sobre todo, a sanear las teorías expurgándolas de hipótesis o explicaciones meramente «ad hoc». Alli donde varias teorías pueden competir entre sí y exhibir parecida capacidad explicativa frente a un cúmulo de datos empíricos ya adquiridos, el único modo de resolver entre ellas es someter a contraste sus, predicciones en una situación experimental crucial y dirimente. La predicción viene a sustituir así a la explicación, o a constituir, al menos su más tangible operacionalización.

Metodológicamente muy fructífero, además de acorde con el talante pragmático de muchos investigadores de la conducta, el criterio de la predicción exacta puede imponerse hasta el punto de relegar a todo principio explicativo no convalidado por exactas predicciones al limbo de lo especulativo y metafisico. En último extremo, dentro de esta orientación, el psicólogo no tiene por qué explicar conductas; se limita a descri- 
birlas y a predecirlas, acaso a tratar de controlarlas. Esta predicción, o bien torna ociosa cualquier explicación, o bien constituye la única explicación científica de la conducta. Es un programa de trabajo que fijó ya Watson (1913) al definir la psicologia desde un enfoque conductista: «La psicología, tal como la ve el conductista, es una rama netamente objetiva y experimental de la ciencia natural. Su meta teórica es la predicción y control de la conducta.» Estas dos líneas primeras del manifiesto conductista no omiten por azar o por olvido la explicación; discurren deliberadamente a su lado, sin entrar en ella.

\section{La explicación probabilística}

Tampoco este último concepto de explicación choca por necesidad con los anteriormente reseñados, y puede muy bien componerse con algunos de ellos. El carácter probabilístico de una explicación se relaciona con la naturaleza también probabilistica de las leyes en que se fundamenta. El léxico a este respecto no está del todo fijo, pero los procesos y leyes basados en la probabilidad suelen contraponerse a los procesos estrictamente nomológicos o determinísticos, asentados en la necesidad o forzosidad de las relaciones. A diferencia de las leyes determinísticas que explican acontecimientos individuales, las probabilísticas sólo explican conjuntos resultantes de la agregación de estos sucesos individuales. El atributo de probabilístico o estocástico puede, por otra parte, resultar aplicado sea a los procesos objetivos estudiados en la ciencia, sea a las proposiciones con que la ciencia representa y explica tales procesos. Las explicaciones, ya nomológicas, ya deterministas de la conducta no acostumbran a recoger esta distinción, $\mathrm{y}$ asumen más bien axiomáticamente que, si nuestros enunciados sobre procesos conductuales son probabilísticos, es porque lo son también los procesos objetivos mismos; y que otro tanto sucede en el contrario caso de que sean nomológicos, a saber, que por igual han de serlo los procesos reales y las proposiciones cientificas acerca de ellos. En psicología apenas se han contemplado explícitamente dos posibilidades, un tanto paradójicas, consideradas por los epistemólogos (cf. Bunge, 1969): la de que, siendo nomológicas o determinísticas las relaciones entre hechos de conducta, a nuestros ojos, a ojos del investigador, y en el estado actual de conocimientos, no puedan aparecer y, por tanto, enunciarse sino como probabilísticas; y la de que, a la inversa, el formato nomológico no se halle más que en la apariencia más superficial de la realidad $\mathrm{y}$, por consiguiente también en nuestras construcciones mentales, como sobrehaz macroscópica determinista que, por agregación, adquieren ante nuestra percepción unos fenómenos que microscópicamente son indeterministas, probabilísticos.

La explicación probabilística de la conducta no se confunde con la adopción de modelos matemáticos. Algunos de éstos, como el de Hull $(1943,1951,1952)$, son decididamente nomológicos, y de ello es plenamente sabedor Estes (1959), cuando presenta su propio modelo estocás- 
tico de aprendizaje en contraposición al modelo hulliano determinístico (y también a otros modelos probabilísticos, como el de Bush y Mosteller, 1951). En la formalización de los fenómenos perceptivos nos encontramos igualmente con modelos matemáticos de diversa naturaleza, ya nomológicos, como en la teoría del nivel de adaptación de Helson (1964), ya probabilísticos, como en la explicación probabilistica, como en el funcionamiento probabilista de Brunswick, y la suposición de que las leyes conductuales obedecen a procesos estocásticos cruza transversalmente a la modelización matemática y en ningún modo coincide con ésta.

En cuanto al fondo de la cuestión, es el conductismo skinneriano el que con mayor energía se ha opuesto a una intelección sólo probabilística de las leyes que rigen la conducta. Su versión de un análisis psicológico causal-funcional (tipo 4) excluye los modelos estocásticos y probabilísticos de explicación. De esta versión hay una firma apología en Sidman (1960), para quien todo enunciado probabilístico sobre fenómenos de la naturaleza y de la conducta es un enunciado tan sólo aproximativo, provisionalmente acaso irremediable por las limitaciones de nuestro conocimiento, pero que puede y debe ser superado en proposiciones más precisas y rigurosas que reflejen relaciones deterministicas y no de simple probabilidad. En el extremo opuesto está una tradición que arranca de Brunswick $(1947,1955,1956)$ y que, a partir de la naturaleza supuestamente probabilistica de la actividad perceptual, postula para toda ciencia, y no sólo para la psicología, un diseño «representativo» de investigación, basado en la inferencia (cf. también Delclaux, 1980, sobre el carácter probabilístico de la inducción en la explicación psicológica).

\section{Conclusión}

No ha sido mi propósito discutir y valorar los diferentes géneros de explicación de la conducta con alguna vigencia en la psicología actual, sino sencillamente exponerlos y tipificarlos. Nuestro conocimiento científico de la conducta, por otra parte, está todavia demasiado verde como para permitirnos el lujo de descartar alguno de ellos como carente de significado o perjudicial para guiar la investigación empírica. La observación explícitamente formulada al caracterizar los dos últimos tipos vale en alguna medida, aunque menor, para los demás: un género de explicación no necesariamente excluye a todos los restantes y puede, al contrario, combinarse muy bien con alguno de ellos - no con todos, esto es no menos cierto - en el marco de una determinada teoría. Una explicación, por ejemplo, puede a la vez ser correlacional, con acentos predictivos, probabilística y formalizada en modelos matemáticos. Una explicación genética es perfectamente compatible con una explicación reduccionista a niveles neurofisiológicos. La naturaleza misma de cada explicación determina las posibles combinaciones de sus compatibilidares e incompatibilidades con otros tipos de explicación y no hace falta acer aqui expreso elenco de tales combinaciones. 
Seguramente algunas conceptualizaciones de la explicación psicológica merecen serias reservas, y en la actualidad (cf. Pinillos, 1980) no se ahorran ya las criticas a la versión fisicalista y mecanicista de la explicación causal. Por mi parte, y tras hacer mención de la circunstancia de que esta explicación, no menos que otras, es susceptible de valoración crítica o, sencillamente, de crítica pura y simple, prefiero orientar mis conclusiones por el camino de la no exclusión de unas explicaciones por otras. Esta orientación responde no al espíritu de componenda propio de un ingenuo eclecticismo, sino a un reconocimiento, primero, de la multiplicidad de disciplinas dentro de la ciencia psicológica, y también, en segundo lugar, de la multiplicidad de los órdenes de explicación de los procesos conductuales.

La pluralidad de posibles explicaciones en psicología se corresponde, por de pronto, con la pluralidad de las disciplinas psicológicas (y no sólo o no tanto de las teorías psicológicas). Cada disciplina tiende a segregar su propio género de explicaciones; o, si se toma la relación a la inversa, cada género de explicación reclama la constitución y desarrollo de la correspondiente disciplina psicológica. La elaboración y sistematización de modelos explicativos matemáticos ha terminado en el desglose disciplinar de una psicología matemática. La perspectiva propia de la psicofisiologia favorece explicaciones reductoras de los procesos conductuales a procesos neurofisiológicos. La de psicología evolutiva propicia explicaciones genéticas. La actual psicología cognitiva se entiende de maravilla con la explicación mediante simulación en computadora. $\mathrm{Y}$ así sucesivamente.

El concepto capaz de abarcar esta pluralidad de explicaciones es el de sobredeterminación. Los fenómenos de conducta se hallan sobredeterminados, o múltiplemente determinados. El hallarse determinados por una génesis evolutiva no impide que también lo estén por unos procesos fisiológicos o por sus dependencia funcionales respecto a ciertos estimulos; ni tampoco obsta a que esa determinación sea probabilística, o también susceptible de simulación en computadora. La pluralidad de modos de explicación en psicología deriva de la sobredeterminación de los fenómenos conductuales. De acuerdo con esta conceptualización, si algún género de explicación debe desde luego ser excluido de la psicologia es cualquier explicación que presuma ella misma de ser exclusiva, o que pretenda explicar monocausalmente los hechos de conducta. Consiguientemente, esta presunción puede que constituya el aspecto más inaceptable de las explicaciones reduccionistas y de las causales -mecánicas, ciertamente insostenibles cuando se presentan - como de hecho acostumbraron presentarse - pretendiendo monopolizar la clave explicatoria de la conducta. Frente a cualesquiera pretendidos monopolios, la multiplicidad de disciplinas psicológicas representa el apropiado correlato y la adecuada garantía epistemológica del principio de la sobredeterminación de la conducta. No es el menor de los méritos de este principio el impedir el prematuro cierre de la teoría y la investigación psicológica, $y$, por el contrario, más bien incitar a exploraciones siempre nuevas en las más diferentes, que no disparatadas, direcciones. 


\section{Referencias}

Abelson, Robert, P. Simulation of Social Behavior. En: Gardner Lindzey/Elliot Aronson, The Handbook of Social Psychology. vol. II. Reading/Massachussets: Addison-Wesley, 1968.

ARDila, Ruben. Psicología fisiológica. México: Trillas, 1975.

Arnau Gras, Jaime. Utilización de modelos matemáticos en Psiclogía. Anuario de Psicología. 1977,17 (2), 3-18.

Aronson, Elliot y Carlsmith, J. Merrill. Experimentation in Social Psychology. En: Gardner Lindzey/Elliot Aronson, The Handbook of Social Psychology, vol. II Reading/Massachussets: Addison-Wesley, 1968.

B.ARRETT, B. H. Behavior analysis. En: J. Wortis (ed.) Mental Retardation and Developmental Disabilities, vol. 9. NewYork: Brunner and Mazel, 1977, pp.141-202.

Binswangre, Ludwig. Articulos y conferencias escogidas. Madrid: Gredos, 1973.

Bjork, RoBER A A. Why mathematical models? American Psychologist, 1973, 426-433.

Bowers, KinnFth S. Situationism in psychology: an analysis and a critique. Psychological Review, 1973, 307-336

Braithaite, R. B. Scientific explanation. Cambridge: Cambridge Univ. Press, 1953. (La explicación cientifica, Madrid: Tecnos, 1965).

Bronfrenbrenner, Urie. The ecology of buman development. Cambridge, Mass.: Harvard University Press, 1979.

BRUNSWIK, E. Systematic and representative design of psychological experiments. Berkeley: University of California Press, 1947.

BRUNSWIK, E. Representative design and probabilistic theory in functional psychology. Psychological Review. 1955, 62, 193-217.

BRUnswik, E. Perception and the representative design of psychological experiments. Berkeley: University of California Press, 1956.

Bunge, MARIO. La investigación cientifica. Barcelona: Ariel, 1980 (ed. original: 1969).

Bunge, Mario. Teoria y realidad. Barcelona: Ariel, 1975 (ed. original: 1972).

Bush, R. R. y Mosteller, F. A. A mathematical model for simple learning. Psychological Review, 1951, 58, 313-323.

Castorina, J. A., Giacobbe, J., Ricco, G. y Plon, M. Explicación y modelos en psicologia. Buenos Aires: Nueva Visión, 1973.

CATTELl, RAYmond B. Personality theory growing from multivariate quantitative research. En: Sigmund Koch, Psycbology: A Study of a Science, vol. III. New York: McGraw-Hill, 1959.

CatTell, R. B. (ed.) Handbook of Multivariate Experimental Psycbology. Chicago: Rand McNally, 1966.

Colby, K. M. Computer simulation of a neurotic process. En: S. Tomkins y S. Messick (eds.) Computer simulation of personality. New York: Wiley, 1963.

COLBY, K. M. Artificial paranoia: A computer simulation of paranoid processes. New York: Pergamon, 1975.

Colby, K. M. Mind models: An overview of current work. Matbematical Biosciences, 1978, 39, 159-185.

CRICK, F. H. C. Reflexiones en torno al cerebro. Investigación y ciencia, 1979, 38: El cerebro, 164-176.

Cronbach, LeE J. The Two Disciplines of Scientific Psychology. American Psychologist, 1957, 671-684.

Cronbach, L. J. y MEeht, P. E. Construct validity in psychological tests. Psychological Bulletin, 1955,52, $281-302$

Delclaux, Isıdoro. Inducción probabilística y explicación psicológica. Análisis y modificación de conducta, $1980,6,11-12,139-145$

Ellenberger, HenRy F. A clinical introduction to psychiatric phenomenology and existential analysis. En: R. May, E. Angel y H. F. Ellenberger (eds.). Existence: $A$ nen dimension in Psychiatry and Psycbology. New York: Basic Books, 1958.

ERIKSON, ERIK H. Identity. Youth and crisis. New York: Norton, 1968. (Identidad. Juventud y crisis. Madrid: Taurus, 1980.)

Estre, W. K. The stadistical approach to learning theory. En: Koch, S. (ed.) Psycbology: $A$ study of a scien$c e$, vol. 2, pp.380-491. New York: McGraw-Hill, 1959.

EYSENCK, H. J. The scientific study of personality. Londres: Routledge and Kegan Paul, 1952. (Estudio cientifico de la personalidad. Buenos Aires: Paidós, 1971.)

GALANTER, Eugene H. On the nature of laws in Psychology. En: J. R. Royce (ed.). Toward Unification in Psycbology. Toronto: Univ. Toronto Press, 1970.

GrkCo, P. Epistemologie de la psychologie. En: J. Piaget y otros, Logique et connaissance scientifique. Epistémologie des sciences humaines. Paris: Gallimard, 1967. (Lógica y conocimiento cientifico. Epistemologia de las ciencias bumanas. Buenos Aires: Proteo, 1972.)

Guthrie, Edwin R. Association by Contiguity. En: Sigmund Koch, Psychology: A Study of a Science, vol. II. New York: McGraw-Hill, 1959.

Helson, HARry. Adaptation-level theory: an experimental and systematic approach to bebavior. New York: Harper and Row, 1964.

Hempel, CARL G. Aspects of scientific explanation and otber essays in the philosopby of science. New York: Free Press, 1965. 
HuEsmanN, L. Rowell y Levinger, George. Incremental exchange theory: a formal model for progression in dyadic social interaction. En: L. Berkowitz y E. Walster (eds.) Advances in experimental social Psycbology, vol. 9. New York: Academic Press, 1976.

HuLl, C. L. Principles of bebavior. New York: Appleton-Century-Crofts, 1943.

Hull, C. L. Essentials of behavior. New Haven, Conn.: Yale University Press, 1951.

Hull, C. L. A Behavior System. New Haven: Yale University Press, 1952.

Jaspers, KARL. Psicopatologia general. Buenos Aires: Paidós, 1955 (1.2 ed.: Allgemeine Psychopatbologie, 1913. Eds. revisadas en: $1920,1922,1946)$.

KAPLAN, A. The conduct of inquiry. San Francisco: Chandler, 1964.

Koch, Sigmund. Epilogue. En: Koch, S. (ed.). Psychology. A Study of a Science. New York: McGraw-Hill, 1900.

LEvi-Strauss, Cl. La pensée sauvage. Paris: Plon, 1962. (El pensamiento salvaje. México: Fondo Cultura Económica, 1964.)

LEWIN, K. Field theory in social science. New York: Harper, 1951.

LOCKARD, R. B. Reflections on the fall of comparative Psychology: Is there a message for us all? American Psycbologist, 1971, 26, 168-179.

MadSEN, KARL B. Theories of motivation. Copenhague: Munksgaard, 1959 (4.2 ed.). (Teorias de la motivación. Buenos Aires: Paidós, 1972.)

Mauss, M. Oewres. Paris: Minuit, 1970. (Lo sagrado y lo profano. Barcelona: Barral, 1970.)

Miller, David B. Roles of Naturalistic Observation in Comparative Psychology. American Psycbologist. 1977, 211-219.

Miller, James G. Toward a general theory for the behavioral sciences. American Psychologist, 1955 , 513-531.

MuSSO, J. Ricardo. Falacias y mitos metodológicos de la psicologia. Buenos Aires: Psique, 1970.

Newell, Allen; Shaw, J. C. y Simon, Herbert A. Elements of a theory of human problem solving. Psycbological Review, 1958, 151-166.

Pelechano, Vicente. Modelos básicos de aprendizaje. Valencia: Alfaplus, 1980.

PIAGET, J. Introduction à l'épistémologie génétique (3 vols.). Paris: Presses Universitaires de France, 1950.

PAIGET, J. L'explication en psychologie et le parallélisme psychophysiologique. En: P. Fraisse y J. Piaget (eds.) Traité de psychologie expérimentale, vol. I: Histoire et métbode. Paris: P.U.F., 1963.

PIAGET, J. Le structuralisme. Paris: P.U.F., 1968.

PIAGet, J. Psychologie et epistémologie. Paris: Gonthier, 1970. (Psicologia y epistemologia, Esplugas/Barcelona: Ariel, 1971.)

PIAGET, JEAN y otros. Tendances principales de la recbercbe dans les sciences sociales, vol. I. Paris: Unesco, 1970. (Tendencias principales de la investigación en las ciencias sociales. Madrid: Alianza, 1975.)

Pinillos, José Luis. Observaciones sobre la psicologia científica. Análisis y modificación de conducta, 1980, 6, 13, 537-590.

PrIBRAM, KARL H. Interrelations of psychology and the neurological disciplines. En Sigmund Koch, Psychology: $A$ Study of a Science, vol. IV. New York: McGraw-Hill, 1962.

Rechea, Cristina. El concepto de modelo en psicología. Análisis y modificación de conducta, 1980, 11-12, 109-115.

Rosenberg, Sfymour. Mathematical models of social behavior. En: G. Lindzey y E. Aronson (eds.) Tbe Handbook of Social Psychology, vol. 2. Research Methods. Reading, Mass.: Addison-Wesley, 1968.

SARTRE, J. P. Critique de la raison dialectique. Paris: Gallimard, 1960.

Sidman, Murray. Tactics of scientific research. New York: Basic Books, Inc., 1960. (Tácticas de investigación cientifica. Barcelona: Fontanella, 1978.)

SIDowski, J. B. (ed.) Special issue: instrumentation in psychology. American Psychologist, 1975, 30, 191-468.

SiDowsKi, J. B. y Ross, S. (eds.) Special issue: instrumentation in psychology. American Psychologist, 1969, $24,187-384$.

Silva, Frernando. Análisis funcional de conducta y diagnóstico psicológico. Análisis y modificación de conducta, 1980, 6, 11-12, 347-359.

SkinNer, B. F. Science and Human Behavior. New York: The Macmillan Company, 1953. (Ciencia y conducta bumana. Barcelona: Fontanella, 1969.)

SkinNer, B. F. Cumulative Record. New York: Appleton-Century-Crofts Inc., 1959. (Registro acumulativo. Barcelona: Fontanella, 1975.)

SPENCE, KENNETH W. The methods an postulates of «behaviorim». Psycbological Review, 1948, 55, 67-78.

TOMkins, S. y MEssick, S. (eds.) Computer simulation of personality. New York: Wiley, 1963.

Tous, J. M. Psicología experimental. Barcelona: Omega, 1978.

Turbayne, Colin Murray. The myth of metaphor. 1970. (El mito de la metáfora. México: Fondo Cultura Económica, 1974.)

Turing, A. M. Computing machinery and intelligence. Mind, 1950, 59, 433-460.

WATson, J. B. Psychology as the behaviorist views it. Psychological Review, 20, 158-177, 1913.

WeRner, H. The concept of Development from a Comparative and Organismic Point of View. En: D. B. Harris (ed.) The Concept of Development. Minneapolis: University of Minneapolis Press, 1957. 


\section{Resumen}

Este artículo bosqueja una exposición, no un debate, a propósito de ciertos tipos de explicación propuestos hoy en psicologia: la explicación comprehensiva, la descriptiva, la reduccionista, la causal-funcional, la correlacional, la genética, la explicación mediante modelos (matemáticos, cibernéticos y otros), la probabilística y la predictiva. La pluralidad de disciplinas en psicología se halla en correspondencia con la multiplicidad de modos de explicar la conducta, puesto que cada disciplina lleva consigo su propio tipo de explicación. De la exposición se desprende, como única tesis de toma de partido, la afirmación de un principio de sobredeterminación de la conducta, cuyas consecuencias epistemológicas se expresa en términos de la necesaria pluralidad de los modos de explicación y de las respectivas disciplinas dentro de la ciencia psicológica.

\section{Abstract}

This article is not intended to be a debate but rather an exposition of certain types of explanation put forward today within psychology: the comprehensive explanation, the descriptive, the reductionist, the causal-functional, the genetic, explanations according to modalities (mathematical, cybernetic, and so forth), that based on probabilities and the predictive. The multiplicity of disciplines within psychology corresponds to that of the ways of explaining behaviour since each discipline has its own explanation.

Through the exposition it can be seen that its only engaging thesis consists of the affirmation of a principle of pluridetermination of behaviour, the epitomological consequence of which is expressed in terms of the necessarily plural explanations and of the respective disciplines within the science of psychology.

\section{Resumé}

Cet article esquisse un exposé, pas un débat, à propos de quelques types d'explication en psychologie: explication descriptive, compréhensive, réductive, fonctionelle, corrélationnelle, génétique, par modèles, probabilistique et prédictive. La pluralité des disciplines en psychologie met en lumière la multiplicité des modes d'expliquer la conduite, puisque chaque discipline porte avec soi son propre type d'explication. De l'exposé en résulte, comme seule prise de parti, une thése en appui d'un principe de surdétermination de la conduite, dont la consequence épistemologique s'exprime en termes de la nécessaire pluralite des modes d'explication et des respectives disciplines. 